\title{
Outcome of Lorazepam-assisted Interview and Psychotherapy in a Middle-aged Female Patient with the Chronic Mixed Dissociative Disorder: A Case Report
}

\author{
Vishnuvardhan Gopalakrishnan ${ }^{1}$, Sumanth T Parameshwaraiah ${ }^{2}$, Vidhyavathi Mallyam ${ }^{3}$, Subhashini Shanmugamurthi ${ }^{4}$ \\ Asha Chandahalli Sannappa ${ }^{5}$
}

\begin{abstract}
Dissociation is a neurotic defense mechanism developed against severe emotional distress and excessive anxiety. Dissociative disorder (DD) is not a single entity, but a wide spectrum of disorders with various subtypes. Typically, patients present with any of one subtype, and cases like dissociative motor disorder comes to medical attention sooner. Contrarily here we are discussing a patient who has symptoms of three subtypes, persisting for a prolonged period. As literature regarding chronic psychogenic aphonia is scarce, we are highlighting the case of a 35-year-old woman with 8 months longstanding history of mixed DD (psychogenic aphonia, dissociative amnesia along with trans and possessional state) because of underlying childhood trauma and multiple conflicts with family members. The focus will be on how lorazepam-assisted interview along with psychotherapy (supportive, catharsis, positive suggestion, and resolution of conflicts) works effectively in a chronic mixed DD. Keywords: Catharsis and positive suggestions, Chronic psychogenic aphonia, Dissociative amnesia, Lorazepam-assisted interview, Trans and possession.

The Journal of Medical Sciences (2020): 10.5005/jp-journals-10045-00154
\end{abstract}

\section{INTRODUCTION}

"Hysteria" was the term used for dissociative disorder (DD) way back during the times of Hippocrates, as it was most commonly seen in the female population and the uterus was believed to be etiological, later with advancements in science, the term dissociative disorder was coined. ${ }^{1,2}$ Dissociative disorder could be caused by extremely stressful situations, including abuse, kidnapping, threats of death, and even childhood trauma. ${ }^{3}$ And the severity of this trauma predicts the chronicity of dissociation. ${ }^{4}$ While the dissociative motor and convulsion disorder are the commonest. ${ }^{3,5}$ A middle-aged man with a short 5-day history of dysphonia recovering completely with a lorazepam interview is reported. ${ }^{6}$ A drug-assisted interview can facilitate the recall of memories and promote the integration of dissociative materials or events. ${ }^{4}$

\section{Case Description}

A 35-year-old married woman who was pre-morbidly well-adjusted was brought to the hospital by her husband with complaints of inability to speak for the past 8 months. In the past, the patient had quarreled with him, following which she stopped talking to him. However, the patient had normal verbal conversations with others which gradually decreased and she was unable to speak totally by 10 days. She communicated non-verbally than on and was unable to remember the quarrel. She heard family guests talking among themselves that she is pretending to seek attention. On hearing this patient had an episode of being possessed by a spirit wherein, she showed physical violence against the guest, but currently, she is unable to recall the episode. Although she was able to converse through gestures, hand and lip movements only, she maintained normal biological functions and carried out all the household activities.
${ }^{1-5}$ Department of Psychiatry, RajaRajeswari Medical College and
Hospital, Bengaluru, Karnataka, India

Corresponding Author: Subhashini Shanmugamurthi, Department of Psychiatry, RajaRajeswari Medical College and Hospital, Bengaluru, Karnataka, India, Phone: +91 9148791770, e-mail: subha.rsm@gmail. com

How to cite this article: Gopalakrishnan V, Parameshwaraiah ST, Mallyam V, et al. Outcome of Lorazepam-assisted Interview and Psychotherapy in a Middle-aged Female Patient with the Chronic Mixed Dissociative Disorder: A Case Report. J Med Sci 2020;6(3):49-50. Source of support: Nil

Conflict of interest: None

Initially patient reported that her inability to speak made her anxious and worried for a couple of months, later she no longer worried about it. Currently, she reports no distress regarding the same. The patient denied any kind of stressors but the family members reported significant interpersonal issues with her elder daughter which remained unsolved and also some of her childhood traumatic experiences of separation from her father at the age of 10 years whom she loved the most. On asking her she reports no recollection of the details regarding her father, also some of her personal information like place of birth, her complete name, schooling, and some parts of her childhood after her father's separation.

The patient was taken to multiple temples for magicoreligious treatment as the family members believed the symptoms are because of black magic, none of them yielded improvements. Eight months later, the patient was bought for psychiatric consultation. She was admitted and physical causes were ruled out through 
otolaryngologist opinion (video laryngoscopy_normal mobile vocal cords) and neurological examination as well (normal MRI).

The patient had features of dissociative motor disorder (psychogenic aphonia), dissociative amnesia, and the episode of trans and possession, hence diagnosis of mixed DD was made and she scored 28.57 on Adult Brief Dissociative Experience Scale (DESB)-modified version [DES-B (Dalenberg C, Carlson E, 2010)]. Tablet escitalopram $10 \mathrm{mg}$ was started.

During her stay at the hospital, she was taken up for therapy sessions daily which included lorazepam interviews and psychotherapy. The procedure was explained to the patient and family members, informed consent was taken. The patient was made to lie down on the bed in the therapy room and a lorazepam interview was done using lorazepam, given slow intravenously with a minimum of $2 \mathrm{mg}$ and a maximum of $6 \mathrm{mg}$ till the patient felt relaxed. Each session lasting for 40-45 minutes along with psychotherapy which aimed at catharsis, suggestibility, and resolution of interpersonal conflicts.

During the first session, the main objective was to give an introduction to therapy and build rapport with the patient. In the second session, the patient was made to relive her traumatic event, helping recollect forgotten memories. During the third session, the patient was able to ventilate problems by crying out loud. The next two sessions were focused on the resolution of mental conflicts/interpersonal problems. During the 6th and 7th sessions, "suggestions" were used, like you are a strong person, you can overcome it and encouraged to speak, these sessions were carried out for a week.

Improvement was noticed within a week, the patient started speaking monosyllables. At this point, supportive psychotherapy was initiated where every day she was encouraged to count numbers up to 50, say English alphabets, months of the year. Although the patient had difficulty speaking initially, she started with simple words and within a couple of weeks could speak phrases. Gradually was made to read out paragraphs from magazines which she could do fluently. A score of 18 on adult (DES-B) was obtained on discharge. No much improvement was seen for dissociative amnesia. Psychoeducation of family members was done.

Follow-up reports showed no new episodes of psychogenic aphonia or trans and possession. A major portion of amnesia persisted which is addressed with psychotherapy during every follow-up with minimal improvement. Tablet escitalopram $10 \mathrm{mg}$ OD orally was continued.

\section{Discussion}

Case reports regarding mixed presentations of DDs are very few. Hence, this case report forms an integral part of the DDs jigsaw.

Most of us would have come across DD, presenting with symptoms mostly of a single subtype. ${ }^{4,6}$ In this case, we are dealing with three subtypes of DDs psychogenic aphonia, dissociative amnesia, and an episode of trans and possession the rarest presentation.

Cases of dissociative motor disorders are noticed earlier and present to medical care within a shorter duration. ${ }^{6}$ The chronicity in our case was maintained because of the gains received out of dissociation and lack of medical attention for 8 months. As DD becomes chronic, they are more difficult to treat and our treatment is aimed at the resolution of current symptoms, restoring functionality, memory retrieval, resolve stressors, and prevention of further episodes. ${ }^{7}$

In these cases, the in-patient approach is a better way than outpatient, as it would avoid interference of family and significant other stressors. ${ }^{8}$ Psychotherapy along with lorazepam interview sessions played a major role in the effectiveness of treatment.

\section{Conclusion}

Unusual presentations of DD in terms of multiple subtype symptoms and chronicity and complete resolution of psychogenic aphonia to sessions of psychotherapy along with lorazepam are noteworthy. The majority of symptoms of dissociative amnesia still remain resistant despite multiple psychotherapy sessions.

\section{References}

1. Reddy LS, Patil NM, Nayak RB, et al. Psychological dissection of patients having dissociative disorder: a cross-sectional study. Indian J Psychol Med 2018;40(1):41-46. DOI: 10.4103/IJPSYM.IJPSYM_ 237_17.

2. Allin $M$, Streeruwitz $A$, Curtis V. Progress in understanding conversion disorder. Neuropsychiat Dis Treatm 2005;1(3):205-209.

3. Sikand M, Arshad R, Beniwal RP, et al. Perceived parental style, cognitive style, and resilience in females with dissociative disorder in India. Indian J Psychia 2019;61(2):177-183. DOI: 10.4103/psychiatry. IndianJPsychiatry_404_18.

4. Seo Y, Shin MH, Kim SG, et al. Effectiveness of lorazepam-assisted interviews in an adolescent with dissociative amnesia: a case report. Neur Regenerat Res 2013;8(2):186-190.

5. Chaturvedi SK, Desai G, Shaligram D. Dissociative disorders in a psychiatric institute in India - a selected review and patterns over a decade. Int J Soc Psychia 2010;56(5):533-539. DOI: $10.1177 / 0020764009347335$.

6. ModakT, Singh S, Kumaran S, et al. Lorazepam-assisted interview in a resistant case of functional dysphonia. J Voice 2020;34(5):811.e7-811. e11. DOI: 10.1016/j.jvoice.2019.01.002.

7. Agarwal V, Sitholey P, Srivastava C, et al. Clinical practice guidelines for the management of dissociative disorders in children and adolescents. Indian J Psychia 2019;61(8):1. DOI: 10.4103/psychiatry. IndianJPsychiatry_493_18.

8. Subramanyam A, Somaiya M, Somaiya $M$, et al. Psychological interventions for dissociative disorders. Indian J Psychia 2020;62(2):280-289. DOI: 10.4103/psychiatry.IndianJPsychiatry_ 777_19. 